\title{
Análisis de la oferta de posgrados en comunicación en España: diversidad e importancia creciente de la modalidad en-línea
}

\author{
Nereida Cea y Aida M. de Vicente \\ Facultad de Comunicación. Universidad de Málaga, Calle de León Tolstoi, s/n, 29010 Málaga, España. \\ (Correo-e: nereidacea@uma.es; aidamaria@uma.es)
}

Recibido Jul. 13, 2020; Aceptado Sep. 9, 2020; Versión final Oct. 21, 2020, Publicado Abr. 2021

\begin{abstract}
Resumen
El objetivo de este estudio es analizar la distribución por universidades, la modalidad de docencia, y la tipología de contenidos de los másteres de comunicación ofertados en España. Se tomó como muestra todos los másteres oficiales impartidos y registrados en la base de datos del Registro de Universidades, Centros y Títulos (RUCT) de España. Los resultados muestran que las universidades no presenciales acumulan más de la mitad $(58,5 \%)$ de la oferta de sus grados, mientras que las universidades presenciales representan el $34,3 \%$ del total. Se observa que los posgrados vigentes y acreditados se distribuyen casi por igual entre centros privados (46\%) y públicos $(54 \%)$, no ocurre así con los títulos propios. Las universidades privadas ofrecen el $75 \%$ de los títulos propios. Se concluye que existe una importancia creciente de las universidades privadas en la oferta en posgrados en comunicación, especialmente con un peso significativo en la modalidad virtual (online) y en el enfoque profesionalista de los contenidos.
\end{abstract}

Palabras clave: periodismo; comunicación; docencia; educación; máster

\section{Analysis of postgraduate communication degrees offer in Spain: diversity and growing importance of online modality}

\begin{abstract}
The primary objective of this research study presents is to examine the distribution, teaching modality, and content of the master's in communication degrees offered by Spanish universities. Registry data for all official masters in communication degrees are retrieved from Spain's official university registry, centers, and degrees (RUCT, in Spanish). The results show that online universities account for more than half (58.5\%) of all masters in communication degrees offered. Traditional (in-person) universities only offer $34.3 \%$ of all degrees. In addition, postgraduate communication degrees are nearly equally distributed between private $(46 \%)$ and public (54\%) universities. However, private universities offer $75 \%$ of all owned and offered degrees by the same institution. It is concluded that there is a growing importance of private universities for the offer of postgraduate communication degrees, especially for degrees that make significant emphasis on virtual learning and that have content focused on professional development.
\end{abstract}




\section{INTRODUCCIÓN}

El contexto en el que se realiza este estudio coincide con una situación de auge de nuevos centros, tanto públicos como privados, que ponen en marcha nuevas titulaciones y cursos de posgrado en Comunicación en España en respuesta a la creciente demanda de esta tipología de estudios. Se considera como punto de partida que más de la mitad de los alumnos de grado en Periodismo y Comunicación que optan por la universidad privada escogen la modalidad on line, de acuerdo a los últimos datos disponibles, que hacen referencia al curso 2015- 2016. Concretamente en estos estudios, un total de 2.440 alumnos optaron por la modalidad no presencial, lo que equivale al $58 \%$ de los alumnos matriculados que estudian en centros privados (tabla 1). Dentro de la modalidad no presencial, la Universitat Oberta de Catalunya es el centro que acapara la mayor parte de los estudiantes de Comunicación, concretamente el $63 \%$ del total. Sin embargo, previsiblemente la Universidad Internacional de la Rioja, debido a una fuerte estrategia de posicionamiento podría haber mejorado esta cuota en los últimos años. Tomando como referencia este punto de diagnóstico, se considera relevante conocer cómo se adapta la oferta de estudios de posgrado con el objetivo de evaluar y prever la evolución que, a la vista de los estudios de grado, podrían seguir los estudios de máster.

El objetivo de este trabajo de ámbito descriptivo es realizar un análisis y diagnóstico de la oferta de másteres en el área de las Ciencias de la Comunicación en España. Con respecto a este objetivo, se consideró como hipótesis que de acuerdo a los rasgos más característicos del sistema universitario son las universidades públicas con modalidad presencial las que presentan una mayor oferta de estudios de posgrado, aunque la evolución es que debido a los cambios que experimenta la sociedad, cada vez sean más demandados las distintas modalidades mediadas tecnológicamente, que van desde opciones de semipresencialidad a modalidades virtuales (síncronas o asíncronas). Es en ese ámbito donde se observa que la universidad privada cuenta con una mayor oferta actualmente, lo que la sitúa en una posición más idónea para adaptarse a la demanda del mercado.

Los objetivos de investigación que se plantean en este trabajo son: O1. Cuantificar y establecer la distribución de los estudios de máster en el área de la Comunicación, de acuerdo a la clasificación de universidades propuesta (presenciales y no presenciales). O2. Describir los másteres universitarios en el área de Comunicación, de acuerdo a parámetros de acreditación, tipología de estudios. O3. Análisis de la modalidad de docencia de los posgrados ofertados en la Universidad española en el área de Comunicación. Este trabajo se enmarca en la línea de otras investigaciones que abordan la estructura de los estudios de grado y posgrado. Destacamos el trabajo realizado por Tejedor \& Cervi (2017) en el que analizan los estudios de Periodismo y Comunicación en las principales universidades del mundo, seleccionando una muestra de una docena de grados a los que aplican para su análisis una plantilla en la que se recogen aspectos básicos como los objetivos del estudio, competencias, estructura del plan, datos generales de las asignaturas.

López García (2010) realiza un estudio comparativo de los estudios de Comunicación de Portugal, Brasil, España, y señala que hay algunas coincidencias en lo que respecta a la evolución que experimentan estos estudios en los distintos países. Se incentiva, por tanto, el intercambio de información y seguimiento de las experiencias en marcha en estos estudios. Y en este sentido, la mejor colaboración pasa, añade el autor referido, "por estrechar las vías de diálogo para conocer mejor los modelos (...) especialmente de aquellos centros que evalúan sus titulaciones, se someten a controles externos nacionales e internacionales y alcanzan el grado de excelencia". Por su parte, Castillo, Nobell \& Muñiz (2013) analizan la oferta de programas de posgrado en comunicación en España. El trabajo aquí realizado supone una actualización del referido estudio y al de Aguilera-Jiménez y Gómez del Castillo (2004), siguiendo una metodología similar, aunque centrando en el análisis de la evolución experimentada desde entonces en los estudios de posgrado y el papel que actualmente están jugando las universidades privadas y la modalidad on line. Esta última cuestión, no ha sido analizada en detalle, por lo que el trabajo aporta elementos de análisis novedosos.

Tabla 1: Número de estudiantes matriculados en U. Privadas, por ámbito de estudio, sexo y universidad. Estudios de Grado y $1^{\circ}$ y $2^{\circ}$ Ciclo. Datos tomados de Ministerio de Educación, Cultura y Deporte.

\begin{tabular}{|l|c|c|}
\hline & Total Universidades & Periodismo y Comunicación \\
\hline Total Universidades Privadas & 173.381 & 5.830 \\
\hline Total Universidades Privadas Presenciales & 125.789 & 3.390 \\
\hline Total Universidades Privadas No Presenciales & 47.592 & 2.440 \\
\hline A Distancia de Madrid & 3.334 & 259 \\
\hline Internacional de La Rioja & 11.432 & 630 \\
\hline Internacional Isabel I & 2.524 & \\
\hline Internacional Valenciana & 928 & \\
\hline Oberta de Catalunya & 29.374 & 1.551 \\
\hline
\end{tabular}


Diversos trabajos evalúan el grado de satisfacción de estudiantes y profesores en entornos de docencia on line, mostrando el alto grado de satisfacción, especialmente entre los estudiantes mayores y en los estudios de máster (Arbaugh, 2004; Akkoyunlu \& Soylu, 2008; Waha y Davis, 2014; Van Tonder \& Steyn, 2018; Ivanova \& Vishnekov, 2020).

El uso de las tecnologías de la información y la comunicación en la docencia mediada tecnológicamente responde en gran parte al mismo avance de la sociedad, que adopta el uso de smartphones y tablets. Efectivamente, la adopción de la tecnología por parte de la sociedad y en especial entre los estudiantes es un factor que está incidiendo en el crecimiento que experimenta la docencia on line (Raghunath, Anker, Nortcliffe, 2018). En este sentido, los procesos de docencia y aprendizaje mediados tecnológicamente requieren de una apuesta por soluciones tecnológicas que faciliten la interacción, hasta el punto de que gran parte de la percepción de éxito de un programa puede depender del uso de soluciones tecnológicas innovadoras que propicien la comunicación (Yadegaridehkordi et al. (2015), y especialmente la interacción (Blankson y Kyei-Blankson, 2008; Díaz y Entonado, 2009). Una de las metodologías innovadoras que experimentan con el uso de soluciones tecnológicas es la modalidad de clase inversa o fliped classroom (Jenkins et al., 2017; Nedeva et al., 2019)

Asimismo, esta investigación enlaza con trabajos previos, como el de Magalhães (2017), que también aborda los estudios de posgrado en comunicación, desde un enfoque cualitativo para describir el diseño curricular y contenido de estos estudios; y con el trabajo de Sierra \& Cabezuelo (2010), en el que se analizan los recursos con los que cuentan las facultades de Comunicación para la formación en competencias en el marco del Espacio Europeo de Educación Superior. Con el mismo enfoque metodológico, aunque analizando los Trabajos Fin de Grado, Sierra et al. (2018), ofrecen un análisis panorámico de la oferta en las distintas Universidades y del ámbito competencial de estos estudios (Zumaquero, 2015). Por su parte, Romacini (2014) amplia el espectro de estudio y establece un marco comparativo para los estudios de posgrado en Comunicación en Iberoamérica. Otra cuestión que se observa del estudio comparativo de países de Latinoamérica, Portugal y España es cómo esta última presenta una mayor oferta de estudios de posgrado con un enfoque profesional y una mayor presencia de oferta on line.

\section{MÉTODO}

Para el análisis de la oferta de enseñanzas universitarias de nivel MECES 3 (EQF Level 7), concretamente máster universitario (60-120 ECTS) en el campo de la Comunicación, se tomó como muestra todos los másteres oficiales impartidos por las universidades españolas, tanto públicos, como privados, e indistintamente de la modalidad de docencia.

La información se tomó de la base de datos del Registro de Universidades, Centros y Títulos (RUCT), elaborado por el Ministerio de Educación, Cultura y Deporte de España. El RUCT es un instrumento en el que se recoge la información actualizada relativa al sistema universitario español. En esta base de datos se pueden consultar los datos relevantes relativos a Universidades, Centros y Títulos. El análisis se realiza en 2019, con datos del curso académico anterior. A partir de esta base de datos, se determina el marco muestral, esto es, las unidades muestrales, constituida por los másteres oficiales, impartidos por las Universidades españolas, en el área de la Comunicación. Para ello se revisan todas las Universidades, Centros y Títulos que imparten títulos oficiales de posgrado (másteres) en el área de Comunicación y de acuerdo a esa selección de estudios dentro del registro del Ministerio, el resultado es una base de la muestra de 163 enseñanzas universitarias de máster, que se imparten entre las 46 universidades españolas, distribuidas entre centros públicos y privados. La información obtenida del Registro de Universidades se contrasta con la información ofrecida por el mismo centro en su página web. El objetivo fue descartar aquellos estudios que, pese a ver recibido la acreditación que les habilita para ser impartidos, no están siendo ofertados por las distintas universidades. De esta forma, del universo que conformaban inicialmente las unidades muestrales que iban a ser objeto de estudio, se descartaron 56 títulos, por lo que finalmente fueron 107 títulos los que conforman la muestra.

Una vez determinado el procedimiento de selección de los elementos muestrales, se diseñó una matriz de análisis para la recogida de datos. La ficha se elabora para cada título y contiene información referente, entre otras, a las siguientes cuestiones: identificación de la universidad o centro que expide el título; tipo enseñanzas conducentes al título (presencial, semipresencial, no presencial); número de plazas reservadas a alumnos de nuevo ingreso. Las variables incluidas en la matriz de análisis se corresponden con los objetivos establecidos en la investigación.

Para completar los datos de la matriz (01 y O2) se realizó un análisis de enfoque cualitativo de los másteres universitarios ofertados en el área de Comunicación a partir de los datos ofrecidos por las mismas Universidades (O3). Para ello se cruzan los datos que aparecen en las páginas web de las distintas 
universidades y facultades, con el Registro de Títulos del Ministerio (RUCT). Así, además de la información obtenida de la base de datos del RUCT, esta se completa con información referente al propio título obtenida de la Universidad donde se imparte. Esta información incluye el idioma de docencia que se utiliza e indicadores de calidad del título, tales como especificación del claustro de profesores y/o especialistas; detalle del plan de estudios y otras cuestiones relevantes.

Asimismo, para la fase de documentación de este trabajo, se analizan y estudian los siguientes materiales: documentos y normativa referente al Espacio Europeo de Educación Superior; publicaciones de la Agencia Nacional de Evaluación de la Calidad y Acreditación (ANECA) sobre los Grados en Ciencias de la Comunicación (Libro Blanco); Informe sobre el estado de la evaluación externa de la calidad en las Universidades Españolas, elaborado por la ANECA; y registro de Universidades, Centros y Títulos.

\section{RESULTADOS}

Uno de los aspectos que se analizan en este trabajo es la distribución de los estudios de posgrado en Comunicación en las distintas Universidades. Para ello, se considerará la diferenciación entre Universidades presenciales y universidades no presenciales. Dentro del primer grupo, se incluyen todas aquellas tanto públicas, como privadas, cuyo modelo principal es la docencia presencial, aunque impartan también, pero de forma minoritaria cursos en distintas modalidades. Dentro del grupo de las Universidades no presenciales se incluyen la Universidad Isabel I, la Universidad Nacional de Educación a Distancia (de naturaleza pública), la Universitat Oberta de Catalunya, la Universidad a Distancia de Madrid, que empezó a impartir docencia el curso 2008-2009, y la Universidad Internacional de La Rioja, que comenzó poco tiempo después. Todas ellas son de naturaleza privada, a excepción de la Universidad Nacional de Educación a Distancia (en adelante, UNED). Hay que precisar que la Universitat Oberta de Catalunya cumple algunas condiciones que la diferencian del resto de universidades no presenciales, ya que pertenece al sistema universitario público catalán y español, pero su modelo es de universidad pública con gestión privada. Actualmente, además de la oferta no presencial de las universidades referidas, muchas otras universidades presenciales públicas y privadas, amplían su oferta con cursos que combinan la docencia presencial, con la semipresencial y la modalidad on line.

En lo que respecta al 01 , se constata que, dentro del área de enseñanza de las Ciencias Sociales, las Universidades no presenciales acumulan más de la mitad de la oferta de sus grados (58,5\%), mientras que, los estudios en esta área en las universidades presenciales representan el 34,3\% del total. Hasta el momento, la universidad pública no ha prestado tanta atención a la modalidad on line y son sólo algunas universidades, por tanto, de forma excepcional, las que ofertan cursos de posgrado a distancia. Según los datos obtenidos en este estudio, en el curso 2017-2018, un total de 26 Universidades públicas en España y 20 privadas ofertaban cursos de posgrado en el área de Comunicación. La distribución por comunidades autónomas muestra que la mayor concentración -tanto de centros públicos, como privados, que imparten estudios de posgrado- se produce en Andalucía (siete universidades públicas, una privada); seguida de Catalunya (seis públicas y cinco) y Madrid (cuatro públicas y seis privadas).

Por lo tanto, en referencia al $\mathrm{O} 1$ se concluye que son las universidades públicas las que tienen una mayor oferta de estudios de posgrado. La mayor concentración de la oferta, tanto pública, como privada, se produce en Andalucía, seguida de Catalunya y Madrid. Los resultados del O2, esto es, del análisis de las características de los másteres universitarios en el área de Comunicación se concretan en los siguientes aspectos identificadores (O2.1) distribución de acuerdo a la tipología de universidad, (O2.2) acreditación de los estudios, (O2.3) tipología de estudios, (O2.4) programación docente, (O2.5) perfil del profesorado.

El número total de cursos de máster universitario acreditados por la ANECA en el área de Comunicación ascendió en 2018 a 107 enseñanzas universitarias de máster (tabla 2). Además, se ofertaron un total más de 28 títulos propios, entre las 46 universidades analizadas, distribuidos entre centros públicos y privados (O2.1.). Cabe destacar como aspecto que merece una consideración detenida que se detectaron un total de 56 programas de posgrado acreditados por la ANECA y, por tanto, que aparecen en el Registro de Universidades, Centros y Títulos (RUCT), que pese a haber sido verificados, no se están impartiendo en sus centros correspondientes. Este dato es relevante, pues supone una cantidad equivalente al $34,35 \%$ del total de los cursos y del $52,33 \%$ de los que sí se están impartiendo con éxito. Estos 56 programas de másteres se corresponden con títulos que han seguido todo el proceso de acreditación y que finalmente por razones determinadas no se están ofertando. Es, por tanto, la universidad privada la que acumula el $86 \%$ de los títulos verificados sin implantación. Al respecto destacan la Universidad Camilo José Cela, la Universidad Antonio Nebrija, la Universidad Central de Catalunya (VIC), IE University y la Universidad Internacional de la Rioja (O2.2.). Dentro de los estudios de posgrado, se puede diferenciar entre los másteres oficiales y los títulos propios. Al respecto, resulta ilustrativo cruzar los datos de la oferta de másteres oficiales y títulos propios, con los de la tipología de las universidades en las que estos se imparten, esto es, su naturaleza pública o privada. 
Se observa que mientras que los posgrados vigentes y acreditados se distribuyen casi por igual entre centros privados y públicos ( $46 \%$, frente a $54 \%$ respectivamente), no ocurre así con los títulos propios. Las universidades privadas ofrecen 3 de cada 4 programas de títulos propios, es decir, el 75\% (O2.3).

Tabla 2: Distribución según tipología de Universidad entre posgrados oficiales (acreditados e implantados) y títulos propios. Datos tomados de Ministerio de Educación, Cultura y Deporte.

\begin{tabular}{|l|c|c|c|c|c|c|}
\hline & \multicolumn{2}{|c|}{$\begin{array}{c}\text { No cursos acreditados } \\
\text { impartiéndose }\end{array}$} & \multicolumn{2}{c|}{$\begin{array}{c}\text { No cursos } \\
\text { Títulos propios }\end{array}$} & $\begin{array}{c}\text { No cursos } \\
\text { Acreditados } \\
\text { No implantados }\end{array}$ \\
\hline Total & \multicolumn{2}{|c|}{107} & \multicolumn{2}{c|}{28} & \multicolumn{2}{c|}{56} \\
\hline Total(Públicas) & 58 & $54 \%$ & 7 & $25 \%$ & 8 & $14 \%$ \\
\hline Total(Privadas) & 49 & $46 \%$ & 21 & $75 \%$ & 48 & $86 \%$ \\
\hline
\end{tabular}

Del análisis de las programaciones docentes se observa que, por una parte, se oferta el formato de máster con una orientación académica, en el que en su plan docente destacan las asignaturas metodológicas y relacionadas con la investigación. Por otro lado, se encuentran los formatos de máster de enfoque profesional y con clara vocación a la especialización, por lo que apuestan por un contenido más práctico y subrayan en sus currículos su conexión con el mundo profesional, bien a través de acuerdos con empresas o instituciones, bien por el perfil eminentemente profesional de su claustro. Se observa que el enfoque profesional de los posgrados es más común en las universidades privadas; mientras que el académico (enfocado a la investigación y a la posterior realización de la tesis doctoral), es más frecuente en las homólogas públicas. Sin embargo, en ambos casos parece un aspecto relevante la acreditación del título. La información sobre la acreditación del título aparece en un lugar visible y prioritario de la web de los centros, ofreciendo información complementaria en muchos casos sobre el proceso y resultado de la acreditación (O2.4 y O2.5)

De acuerdo a los datos recogidos en este estudio, se puede afirmar que las Universidades privadas tienden más que las públicas a ampliar su oferta de cursos de posgrados oficiales con un enfoque profesional y más versátil, tanto en su adaptación a las necesidades del mercado, como, si es necesario, a su extinción en caso de ser necesario. Por otra parte, se observa que en la estrategia de promoción estas instituciones ponen de relieve cuando cuentan con la colaboración de alguna empresa. Por ejemplo, el máster en la Universidad Llul tiene un acuerdo de colaboración con el grupo Godó, mientras que la Universitat Abat Oliva, por su parte, cuenta entre sus socios con La Vanguardia. En el País Vasco, la Universidad ofrece un acuerdo de prácticas con el periódico El Correo. Y la Universidad Miguel Hernández imparte su curso de posgrado en colaboración con LENS Escuela de Artes Visuales, además de contar con el patrocinio de las principales empresas del sector audiovisual de la Comunidad Valenciana. Especial mención merece el máster de la Universidad Rey Juan Carlos que ofrece prácticas en Radio Televisión Española y el de la Universidad Francisco Victoria, que dispone de prácticas en TeleMadrid. También cabe destacar el Máster en Comunicación y Periodismo Deportivo por la Universidad Europea de Madrid, que se realiza en colaboración con el Real Madrid y que ha sido elegido entre los 5 mejores másteres especializados en el Área de Comunicación en España. Sin embargo, es la Universidad San Pablo CEU la que tiene una política de colaboraciones más potente, que aseguran prácticas en distintos medios del grupo Unidad Editorial y la COPE.

Por lo tanto, se concluye que del análisis de los estudios de posgrado universitarios en el área de Comunicación se encontraron un total de 107 enseñanzas universitarias de máster y más de 28 títulos propios. A esta oferta se suman 56 títulos que, pese a lograr la acreditación de la ANECA, no se ofertaron, lo que muestra que más del $30 \%$ de los títulos acreditados en el área finalmente no se imparten con éxito. Además, las universidades privadas sobresalen por su oferta de títulos propios, ofertando 3 de cada 4 programas en España. En relación al O3 (Análisis de la modalidad de docencia de los posgrados ofertados en la Universidad española en el área de Comunicación), se observa que un aspecto significativo es que, a pesar del auge de la modalidad on line, solo el $18,69 \%$ de los títulos ofertados se imparten exclusivamente on line (tabla 3). Sin embargo, este porcentaje es muy superior, puesto que un $13 \%$ más incluye distintas variables de semipresencialidad. De acuerdo a los datos obtenidos se imparten de forma exclusiva en modalidad presencial un total de 58 másteres, que equivale al $54 \%$ de la oferta total en España.

Se observa una clara correspondencia entre la modalidad de docencia (presencial, semipresencial y on line) y el tipo del máster, esto es, se constata una relación entre la modalidad de docencia y el enfoque del máster (orientado a la investigación o al ámbito profesional). Mientras que prácticamente la totalidad de los másteres orientados a la investigación son presenciales (con tres excepciones: la Universidad de Granada, Zaragoza y 
Pompeu Fabra, que ofertan másteres de investigación también de forma on line), una amplia mayoría de enfoque profesional se ofertan bajo distintas modalidades que abarcan desde el formato exclusivo on line a una modalidad mixta en la que el alumno puede escoger entre presencial, semipresencial y a través de internet, con las distintas variantes de combinaciones, tal y como se detalla en la figura 1.

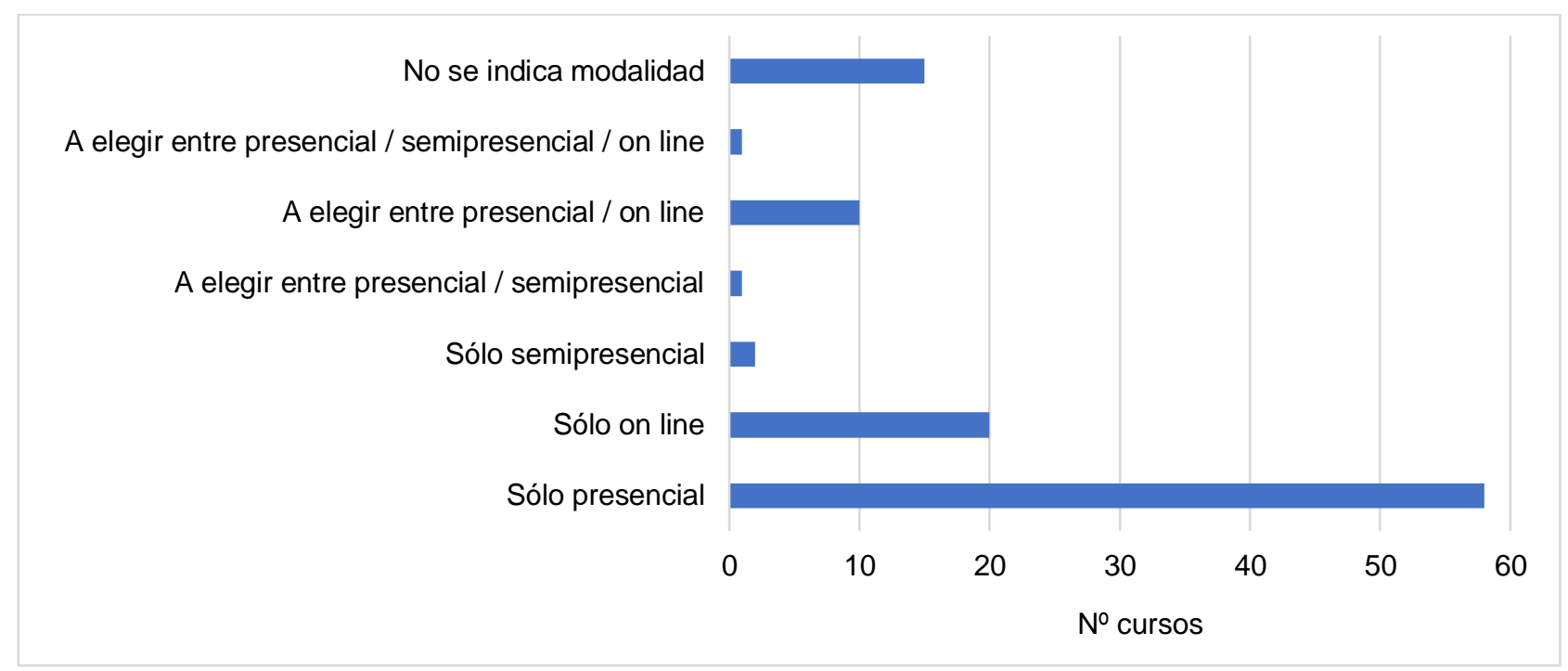

Fig. 1: Tipología de los cursos de posgrados en Comunicación ofertados según modalidad. Datos tomados de Ministerio de Educación, Cultura y Deporte.

Son nuevamente los centros privados, esto es, la Universitat Oberta de Catalunya, la Universidad Internacional de la Rioja, la Universidad a Distancia de Madrid y Antonio Nebrija, las que acaparan prácticamente la totalidad de los másteres en Comunicación en la modalidad on line. Además, cabe señalar la limitada oferta de la UNED, que se concreta en tres cursos de posgrado en el área de Comunicación, a pesar del amplio abanico de cursos que ofrece en otras múltiples disciplinas. Por lo tanto, de acuerdo al $\mathrm{O} 3$ (Análisis de la modalidad de docencia de los posgrados ofertados en la Universidad española en el área de Comunicación. uno de cada tres másteres se puede cursar on line) se observa que son las universidades privadas (especialmente Universitat Oberta de Catalunya, Universidad Internacional de la Rioja, Universidad a Distancia de Madrid y Antonio Nebrija), las que acaparan prácticamente la totalidad de los másteres en Comunicación en la modalidad on line.

\section{DISCUSIÓN}

Como elemento de reflexión prospectivo, cabe añadir que estudios como el aquí realizado parecen pertinentes como puntos de reflexión y análisis de cara a la implantación futura de nuevos estudios de Comunicación. Previsiblemente proseguirá la expansión de la oferta de posgrados en el sistema universitario, tal y como viene ocurriendo en la última década, aumentando así la distancia existente entre los estudios academicistas y los de enfoque profesionalista. En esa dicotomía, los centros que optan por la modalidad on line inclinan su oferta hacia estudios más enfocados a la especialización y profesionalización, que hacia aquellos dirigidos a la formación en investigación. Tal y como se ha constatado en este estudio, aunque en términos generales, en el área de Comunicación las universidades privadas ofrecen un porcentaje minoritario de la oferta de posgrados, no ocurre así en la modalidad de la docencia on line. Dado el crecimiento que esta modalidad está experimentando en los últimos años, y a la luz de los cambios en la sociedad, esta tendencia puede llegar a funcionar como elemento dinamizador de cambios en la institución universitaria.

En este trabajo se analiza la oferta de enseñanzas universitarias con el objetivo de evaluar el estado actual de la Educación Superior en España en el área de los posgrados en Comunicación en España y su potencial para seguir creciendo, especialmente en los países de habla hispana y en la modalidad on line. Las conclusiones fruto de este trabajo sugieren que los rasgos dominantes en la enseñanza superior en España están cambiando de forma gradual debido principalmente al crecimiento de la cuota de las universidades privadas y el crecimiento de la modalidad de impartición on line en los estudios oficiales de posgrado en el área de Comunicación.

Fruto del análisis realizado, se observan dos tipos de másteres muy diferenciados. Por una parte, se encuentra el grupo de estudios orientados a la investigación. Estos estudios son los que permiten acceder al segundo período de investigación del Doctorado. El segundo bloque lo constituyen los másteres orientados a 
la especialización, que se pueden cursar en cualquier momento una vez obtenido el título de grado y que tienen como finalidad un aprendizaje mucho más especializado de la praxis profesional. Mientras que la práctica totalidad de los másteres orientados a la investigación son impartidos en universidades públicas, los posgrados oficiales y títulos propios de especialización son mayormente coto de las privadas. Existen excepciones, como es el caso de la Universidad Carlos III o la Universidad Pompeu Fabra, que ofrece ambos tipos de estudios.

A su vez, también algunas universidades privadas como la Universitat Oberta de Catalunya y la Universidad Internacional de la Rioja están impulsando la formación de investigadores por lo que desde hace unos años vienen ofreciendo másteres enfocados a la investigación, que también tienen su enfoque práctico, por lo que pueden parecer asequibles e interesantes a los dos perfiles de alumnado. El primer aspecto relevante que se observa es que existe una oferta de másteres más amplia de lo que inicialmente se preveía en la hipótesis de partida de este trabajo. Asimismo, existe un número importante de estudios que, pese a estar aprobados por la ANECA, no se están impartiendo. Queremos detenernos en este hecho porque lo consideramos clave para entender el mapa de titulaciones de posgrado en el área de Comunicación.

Es reseñable que 56 cursos de posgrado, lo cual supone un número significativo equivalente a más del $50 \%$ de los cursos que ya se imparten, pese a ver sido evaluados por la ANECA, verificados por el Consejo de Universidades y contar con el informe de autorización de la Comunidad pertinente, no se están impartiendo y, en la mayor parte de los casos, no se han llegado a implantar nunca. Esto sugiere que la oferta por parte de las universidades ha sido mayor que la demanda que ha experimentado el mercado. Si se desglosan esos datos de títulos no implantados según tipología de universidades, se observa que son los centros privados los que acaparan el mayor número de cursos de posgrados verificados y autorizados que no se han llegado a implantar (un total de 48 posgrados, que suponen el $86 \%$ del total). Teniendo en cuenta que el Registro de Universidades, Centros y Títulos (RUCT), fue creado en septiembre de 2008, esto indica que en poco menos de 8 años gran número de nuevos títulos han sido acreditados sin llegar a tener implantación previsible.

El hecho de que los estudios de segundo ciclo acreditados que, pese a figurar en RUCT no se están impartiendo, correspondan en su mayoría a Universidades privadas sugiere que son las universidades privadas, por su propia naturaleza, las que muestran un mayor grado de flexibilidad y adaptación a la demanda, así como una mayor versatilidad a la hora de poner en marcha cursos, tanto oficiales, como títulos propios, más adaptados a las necesidades del mercado profesional y con mayor grado de especialización. Esta adaptación a la demanda del mercado no se observa en la misma medida en las universidades públicas. Sin embargo, podría ser un elemento a tener en cuenta de cara al futuro, en la implantación de nuevos estudios.

\section{CONCLUSIONES}

De acuerdo al trabajo presentado y a los resultados obtenidos, se pueden plantear las siguientes conclusiones principales:

1.- La oferta de estudios de posgrado en el área de enseñanza de las Ciencias Sociales en España es mayor en la modalidad de docencia no presencial (58,5\% de los títulos), frente a la no presencial (34,3\%). Además, el resto se completa con distintas combinaciones de las modalidades presenciales, semipresenciales y on line. La modalidad on line es más frecuente en los títulos impartidos por las Universidades privadas y son sólo algunas universidades públicas las que incluyen cursos de posgrado a distancia.

2.- En lo que respecta a la distribución de acuerdo a la tipología de universidad, se concluye que un total de 46 universidades analizadas, distribuidas entre centros públicos y privados, ofertaron un total de 107 enseñanzas universitarias de máster y 28 títulos propios.

3.- Un total de 56 programas de posgrado acreditados por la ANECA, a pesar de haber sido verificados, no se están impartiendo, lo que muestra que más del $30 \%$ de los títulos acreditados en el área finalmente no se imparten con éxito.

4.- De acuerdo a la tipología de estudios, se concluye que la oferta de posgrados vigentes se distribuye casi de forma equivalente entre centros privados y públicos (46\%, frente a $54 \%$ respectivamente). Por el contrario, la oferta de títulos propios se concentra en las universidades privadas, que imparten 3 de cada 4 programas de títulos propios (75\%).

3.- Por último, del análisis de las características de los estudios ofertados, se observa que mayoritariamente la universidad pública ofrece el formato de máster de orientación académica, con planes docentes en los que destacan asignaturas metodológicas y relacionadas con la investigación; mientras que los formatos de máster de enfoque profesional y con clara vocación a la especialización se concentran en los centros privados. 


\section{REFERENCIAS}

Aguilera-Jiménez, A. y Gómez Del Castillo, M. T., Perspectivas de la Universidad española en el Espacio Europeo de Educación Superior, Revista Latina de Comunicación Social, 57(7), 1-5 (2004).

Akkoyunlu, B. y Soylu, M. Y., A study of student's perceptions in a blended learning environment based on different learning styles, Journal of Educational Technology \& Society, 11(1) (2008).

ANECA., Informe sobre el estado de la evaluación externa de la calidad en las Universidades Españolas. Madrid, Ministerio de Educación, Cultura y Deporte (2015).

ANECA., Libro Blanco de los Títulos de Grado en Ciencias de la Comunicación. Madrid, Ministerio de Educación, Cultura y Deporte (2005).

Arbaugh, J. B., Learning to learn online: A study of perceptual changes between multiple online course experiences, https://doi.org/10.1016/j.iheduc.2004.06.001, The internet and higher education, 7(3), 169-182 (2004).

Blankson, J., y Kyei-Blankson, L., Nontraditional students' perception of a blended course: Integrating synchronous online discussion and face-to-face instruction, Journal of Interactive Learning Research, 19(3), 421-438 (2008).

Castillo, A., Nobell, A. y Muñiz, J., EEES y la formación en comunicación en España. La estructura de la oferta de posgrado, https://doi.org/10.5209/rev_ESMP.2013.v19.42015, Estudios sobre el mensaje periodístico, 19 (1), $99-110$ (2013).

Díaz, L. A. y Entonado, F. B., Are the functions of teachers in e-learning and face-to-face learning environments really different?, Journal of educational technology \& society, 12(4), 331-343 (2009).

Ivanova, E.M. y Vishnekov, A.V., A computer design method of an effective educational trajectory in blended learning based on students' assessment, https://doi.org/10.1007/s10639-020-10109-3, Education and Information Technologies, 25, 2, 1439-1458 (2020).

Jenkins, M., Bokosmaty, R., Brown, M., Browne, C., Gao, Q., Hanson, J., y Kupatadze, K., Enhancing the design and analysis of flipped learning strategies, https://doi.org/10.20343/teachlearninqu.5.1.7, Teaching \& Learning Inquiry, 5(1), 1$12(2017)$.

López García, X., La formación de los periodistas en el siglo XXI en Brasil, España, Portugal y Puerto Rico, https://doi.org/10.4185/RLCS-65-2010-896-231-243, Revista Latina de Comunicación Social, 65, 231-243 (2010).

Magalhães, L., Cómo analizar los posgrados en comunicación de lberoamérica. Los enfoques y sus implicaciones políticas, Chasqui. Revista Latinoamericana de Comunicación, 136, 279-293 (2017).

Ministerio de Educación, Datos y cifras del sistema Universitario Español. http://www.educacionyfp.gob.es/servicios-alciudadano/estadisticas.html (2018)

Nedeva, V., Dineva, S. y Ducheva, Z., Students in blended learning by flipped classroom approach, https://doi.org/10.33407/itlt.v72i4.3046, Information technologies and learning tools, 72(4), 204-213 (2019).

Raghunath, R., Anker, C. y Nortcliffe, A., Are academics ready for smart learning?, https://doi.org/10.1111/bjet.12532, British journal of educational technology, 49(1), 182-197 (2018).

Romancini, R., Os Estudos em comunicação e educação no espaço Iberoamericano: panorama da pósgraduação, https://doi.org/10.7764/cdi.35.660, Cuadernos. info, 35, 103-117 (2014).

Rowenthal, P., Bauer, C. y Chen, K. Z., Student perceptions of online learning: An analysis of online course evaluations, https://doi.org/10.1080/08923647.2015.1023621, American Journal of Distance Education, 29(2), 85-97 (2015).

Sierra, F., y Cabezuelo, F., Recursos de las facultades de comunicación para la formación en competencias en el marco del Espacio Europeo de Educación Superior, https://doi.org/10.31921/doxacom.n11a2, Doxa Comunicación, 11, 31-54 (2010).

Sierra, J., Libera, S. y Luceño, B., Análisis de la materia Trabajos de Fin de Grado (TFG) en los grados de Ciencias de la Comunicación en España, https://doi.org/10.3989/redc.2018.4.1561, Revista Española de Documentación Científica, 41 (4), e220 (2018).

Tejedor Calvo, S. y Cervi, L., Análisis de los estudios de Periodismo y Comunicación en las principales universidades del mundo, https://doi.org/10.4185/RLCS-2017-1238, Competencias, objetivos y asignaturas, Revista Latina de Comunicación Social, 72, 1.626-1.647 (2017).

van Tonder, S. y Steyn, G. M., Exploring Role Players' Experiences of Learning in Higher Education through a Hybrid Study Approach: A Case Study, Pertanika Journal of Social Sciences \& Humanities, 26(4) (2018).

Waha, B., y Davis, K., University students' perspective on blended learning, https://doi.org/10.1080/1360080X.2014.884677, Journal of Higher Education Policy and Management, 36(2), 172-182 (2014).

Yadegaridehkordi, E., lahad, N. A., y Ahmad, N., User perceptions of the technology characteristics in a cloud-based collaborative learning environment: a qualitative study, https://doi.org/10.1504/IJTEL.2015.071923, International Journal of Technology Enhanced Learning, 7(1), 75-90 (2015).

Zumaquero, L., La acción tutorial en los trabajos de fin de grado: análisis de su funcionamiento en la titulación de grado de derecho de la universidad de Málaga, Docencia y Derecho, Revista para la Docencia Jurídica Universitaria, 9, 1-14 (2015). 